Homology, Homotopy and Applications, vol.4(2), 2002, pp.397-407

\title{
THE RIEMANN TENSOR FOR NONHOLONOMIC MANIFOLDS
}

\author{
DIMITRY LEITES
}

(communicated by Larry Lambe)

\begin{abstract}
For every nonholonomic manifold, i.e., manifold with nonintegrable distribution the analog of the Riemann tensor is introduced. It is calculated here for the contact and Engel structures: for the contact structure it vanishes (another proof of Darboux's canonical form); for the Engel distribution the target space of the tensor is of dimension 2. In particular, the Lie algebra preserving the Engel distribution is described.

The tensors introduced are interpreted as modifications of the Spencer cohomology and, as such, provide with a new way to solve partial differential equations. Goldschmidt's criteria for formal integrability (vanishing of certain Spencer cohomology) are only applicable to "one half" of all differential equations, the ones whose symmetries are induced by point transformations. Lie's theorem says that the "other half" consists of differential equations whose symmetries are induced by contact transformations. Therefore, we can now extend Goldschmidt's criteria for formal integrability to all differential equations.
\end{abstract}

\section{To Jan-Erik Roos on his sixty-fifth birthday}

\section{Introduction}

Let $W_{k}^{n}$ be the space of germs of irreducible $k$-dimensional distributions at the origin of $\mathbb{R}^{n}$. The orbits of Diff $\mathbb{R}^{n}$-action on $W_{k}^{n}$ are usually of infinite codimension. As shown in [VG1], there are 3 types of exceptions: (1) $W_{k}^{n}$ is a point, (2) codimension 1 distributions determined as zero set of $\alpha=0$ for the forms $\alpha$ of maximal class (recall that the class of the form is the invariant equal to the least number of

Partially supported by an NFR grant. I am thankful to A. Vershik and V. Gershkovich who put the problem; to L. Faddeev and A. Reyman and to R. Cianci, U. Bruzzo and E. Massa for hospitality when a guest of the Euler IMI in November 1990 and the Genova University in February 1991, respectively, and where these results were delivered. They were main ingredient of the theoretical part of my ICTP lectures in 1991, cf. [GL1]. I thank P. Grozman who checked my computations with the help of his SuperLie package [GL2].

Received March 1, 2001, revised February 21, 2002; published on July 12, 2002.

2000 Mathematics Subject Classification: 17A70, 17B35

Key words and phrases: Engel distribution, Lie algebra cohomology, Cartan prolongation, Riemann tensor, nonholonomic manifold

(C) 2002, Dimitry Leites. Permission to copy for private use granted. 
variables actually involved)

$$
\alpha= \begin{cases}d t+\sum_{1 \leqslant i \leqslant r}\left(p_{i} d q_{i}-q_{i} d p_{i}\right) & \text { for } n=2 r+1 \\ d t+\sum_{1 \leqslant i \leqslant r-1}\left(p_{i} d q_{i}-q_{i} d p_{i}\right) & \text { for } n=2 r\end{cases}
$$

called (usually, for $n$ odd) a contact structure (see $[\mathbf{Z}]$ ) and (3) the Engel distribution locally given by the system of Pfaff equations $\alpha_{1}=0, \alpha_{2}=0$, where

$$
\alpha_{1}=d x_{4}-x_{3} d x_{1}, \quad \alpha_{2}=d x_{3}-x_{2} d x_{1} .
$$

Any 4-dimensional manifold with the Engel distribution (E) will be called an Engel manifold.

Both contact and Engel manifolds are examples of nonholonomic manifolds (i.e., manifolds with nonintegrable distributions). Recall that the term nonholonomic was coined by $\mathrm{H}$. Hertz $[\mathbf{H}]$ in his attempts to geometrically describe motions in such a way as to eliminate the notion of "force". For a historical review see [VG2]; see also a very interesting paper by Vershik $[\mathbf{V}]$ with first rigorous mathematical formulations of nonholonomic geometry and indications to various possible applications in various, sometimes unexpected, areas (like optimal control or macro-economics). In [V] Vershik summarizes about 100 years of studies of nonholonomic geometry (Hertz, Carathéodory, Vrănceanu, Wagner, Schouten, Faddeev, Griffiths, Godbillon, to name a few; to his list I'd add that MathSciNet returns about 700 entries for nonholonomic, and for synonyms: 70 more for sub-Riemannian,1700 for Finsler, several dozens for "cat's problem" and autoparallel; physics have their own simialr lists with thousands of entries because there seems to be more, actually, nonholonomic dynamical systems than holonomic ones; finally supergravity is also a nonholonomic structure, albeit on supermanifolds - ca 1800 more entries). In [V] Vershik also declares that "it is probably impossible to construct an analog of the Riemann tensor for the general nonholonomic manifold" though in some particular cases of small dimension they had been constructed (Martinet) (and, one can add now, in papers on supergravity).

Here I introduce the invariants that characterize "nonflatness" of nonholonomic manifolds, i.e., positively solve this old problem. This definition appeared around 1989 but publication of this paper was delayed and the definition first (as far as I know) appeared in contetext of supergravity in $[\mathbf{L P}]$. For application to SUGRA (supergravity equations) and other instances where the N-curvature tensors (the left hand side of SUGRA) are calculated see [GL1], [LPS], [LSV].

The general definition is illustrated with contact and Engel manifolds. As expected, for the contact structure these invariants vanish, thus giving one more proof of the Darboux theorem on the canonical form of the contact form; one can show that they vanish for $n$ even as well. The Engel manifolds are more rigid: there could be nonflat ones among them.

Observe that our invariants, though natural analogs of the curvature and torsion tensors, do not coincide on nonholonomic manifolds with the classical ones and bearing the same name. Indeed, by definition on any nonholonomic manifold, there is a nonzero classical torsion while every contact manifold is flat in our sense. To 
avoid confusion, we should always add adjective "nonholonomic" for the invariants introduced below. Since this is too long, we will briefly say $N$-torsion, $N$-curvature, etc.

For manifolds with a $G$-structure, the prototypes of the invariants we consider are well-known: these are structure functions of the $G$-structure [St]; an important particular case is $G=O(n)$ when the structure function is called the Riemann tensor.

A theorem of Lie states that there are two types of differential equations, considered as submanifolds in the jet bundles: (1) those whose symmetries are induced by point transformations and (2) those whose symmetries are induced by contact transformations. For equations of the first type Goldschmidt gave criteria for formal integrability [BCG]. The notions introduced below — generalizations of Spencer cohomology for nonholonomic case - provide with a straightforward generalization of Goldschmidt's criteria for formal integrability to the second half of differential equations.

Finally, observe that "non-flatness" of distributions with non-linear constraints (such as fields of cones $[\mathbf{A}]$, foreseen by Vershik in $[\mathbf{V}]$, or fields of spheres, like the ones constructed by any driver who switched the cruise control ON, can also be tested by invariants I introduce; in the case of the fields of solids, however, the Lie algebras involved are of infinite dimension (they correspond to "curved Grassmannians" of submanifolds, rather than linear subspaces) and feasibility to obtain the final result becomes very doubtful.

The waves in plasma is another example of a nonholonomic system with infinite dimensional algebras involved. This is a pity, since the curvature tensor is known to be responsible for stability of the dynamical system expressed by geodesics; so, in principle, we could just have computed the N-curvature in this case and settle an intriguing question: "is cold fusion possible or not?".

\section{$\S 1$. Structure functions}

Let $M$ be a manifold of dimension $n$ over a field $\mathbb{K}$. Let $F(M)$ be the frame bundle over $M$, i.e., the principal $G L(n ; \mathbb{K})$-bundle. Let $G \subset G L(n ; \mathbb{K})$ be a Lie group. A $G$-structure on $M$ is a reduction of the principal $G L(n ; \mathbb{K})$-bundle to the principal $G$-bundle. Another formulation seems to be clearer: if one can select transition functions from one coordinate patch to another so that they belong to $G$ for every intersection pair of patches, then we have a $G$-structure on $M$.

The simplest $G$-structure is the flat $G$-structure defined as follows. Let $V$ be $\mathbb{K}^{n}$ with a fixed frame. Fix a frame in $V$. Having identified $V$ with $T_{v} V$ via parallel translation along $v$, we get a fixed frame in every $T_{v} V$. The flat $G$-structure is the bundle over $V$ whose fiber over $v \in V$ consists of all frames obtained from the fixed one under the $G$-action.

In textbooks on differential geometry (e.g., in [St]) the obstructions to identification of the $k$-th infinitesimal neighborhood of a point $m \in M$ on a manifold $M$ with $G$-structure with that of a point of the manifold $V$ with the above flat $G$-structure are called structure functions of order $k$. It is also explained there that such an 
identification is possible provided all structure functions of lesser orders vanish.

To formulate the precise statement, we need some definitions. Let $S^{i}$ denote the operator of the $i$-th symmetric power. Set

$$
\mathfrak{g}_{-1}=T_{m} M, \quad \mathfrak{g}_{0}=\mathfrak{g}=\operatorname{Lie}(G) ;
$$

for any $i>0$ and $k=1, \ldots, i$ set:

$$
\begin{aligned}
\mathfrak{g}_{i}= & \left\{X \in \operatorname{Hom}\left(\mathfrak{g}_{-1}, \mathfrak{g}_{i-1}\right) \mid X\left(v_{1}\right)\left(v_{2}, v_{3}, \ldots, v_{i}\right)=\right. \\
& \left.=X\left(v_{2}\right)\left(v_{1}, v_{3}, \ldots, v_{i}\right) \text { for any } v_{k} \in \mathfrak{g}_{-1}\right\} .
\end{aligned}
$$

Now, set $\left(\mathfrak{g}_{-1}, \mathfrak{g}_{0}\right)_{*}=\underset{i \geqslant-1}{\oplus} \mathfrak{g}_{i}$.

Suppose that the $\mathfrak{g}_{0}$-module $\mathfrak{g}_{-1}$ is faithful. Then, clearly,

$$
\left(\mathfrak{g}_{-1}, \mathfrak{g}_{0}\right)_{*} \subset \mathfrak{v e c t}(n)=\mathfrak{d e r} \mathbb{K}\left[\left[x_{1}, \ldots, x_{n}\right]\right] \text {, where } n=\operatorname{dim} \mathfrak{g}_{-1} .
$$

It is subject to an easy verification that the Lie algebra structure on $\mathfrak{v e c t}(n)$ induces same on $\left(\mathfrak{g}_{-1}, \mathfrak{g}_{0}\right)_{*}$. The Lie algebra $\left(\mathfrak{g}_{-1}, \mathfrak{g}_{0}\right)_{*}$ will be called the Cartan's prolong (the result of Cartan's prolongation) of the pair $\left(\mathfrak{g}_{-1}, \mathfrak{g}_{0}\right)$.

Let $\Lambda^{i}$ be the operator of the $i$-th exterior power; set $C_{\left(\mathfrak{g}_{-1}, \mathfrak{g}_{0}\right)_{*}}^{k, s}=\mathfrak{g}_{k-s} \otimes \Lambda^{s}\left(\mathfrak{g}_{-1}^{*}\right)$; we usually drop the subscript of $C_{\left(\mathfrak{g}_{-1}, \mathfrak{g}_{0}\right)_{*}}^{k, s}$ or indicate only $\mathfrak{g}_{0}$. Define the differential $\partial_{s}: C^{k, s} \longrightarrow C^{k, s+1}$ setting for any $v_{1}, \ldots, v_{s+1} \in V$ (as usual, the slot with the hatted variable is ignored):

$$
\left(\delta_{s} f\right)\left(v_{1}, \ldots, v_{s+1}\right)=\sum_{i}(-1)^{i}\left[f\left(v_{1}, \ldots, \hat{v}_{s+1-i}, \ldots, v_{s+1}\right), v_{s+1-i}\right] .
$$

As expected, $\delta_{s} \delta_{s+1}=0$. The homology of this bicomplex is called Spencer cohomology of the pair $\left(\mathfrak{g}_{-1}, \mathfrak{g}_{0}\right)$ and denoted by $H_{\left(\mathfrak{g}_{-1}, \mathfrak{g}_{0}\right)_{*}}^{k}$.

Proposition - ([St] $]$ Structure functions constitute the space of the $(k, 2)$-th Spencer cohomology of the Cartan prolongation of the pair $\left(T_{m} M, \mathfrak{g}\right)$ where $\mathfrak{g}=\operatorname{Lie}(G)$.

The Riemann tensor on an $n$-dimensional Riemannian manifold is the structure function from $H_{\left(\mathfrak{g}_{-1}, \mathfrak{o}(n)\right)_{*}}^{k, 2}$.

Remark . (cf. $[\mathbf{G}]$.)

$$
\underset{k}{\oplus} H_{\left(\mathfrak{g}_{-1}, \mathfrak{g}_{0}\right)_{*}}^{k, s}=H^{s}\left(\mathfrak{g}_{-1} ;\left(\mathfrak{g}_{-1}, \mathfrak{g}_{0}\right)_{*}\right) .
$$

This remark considerably simplifies calculations, in particular, if the Lie algebra $\left(\mathfrak{g}_{-1}, \mathfrak{g}_{0}\right)_{*}$ is simple and finite dimensional: we can apply the Borel-Weil-Bott... (BWB) theorem. In the nonholonomic case considered in what follows the remark provides with a compact definition ${ }^{1}$ of structure functions. We can recover the bigrading (explicitly preserved in $[\mathbf{T}]$ ) at any moment but to work with just one grading is much simpler, as with generating functions of series instead of individual terms. Moreover, there are a few general theorems that help to calculate Lie algebra cohomology and no theorems on Spencer cohomology.

${ }^{1} \mathrm{Cf}$. with the problems encountered in the pioneer paper $[\mathbf{T}]$, where the case $d=2$ for Lie algebras was independently introduced. 


\section{$\S 2$. Structure functions of nonholonomic manifolds}

To embrace contact-like structures we have to slightly generalize the notion of Cartan prolongation: the tangent space to a point of a nonholonomic manifold is naturally rigged with a nilpotent Lie algebra structure (e.g., for any odd dimensional contact manifolds this is the Heisenberg algebra), cf. [VG].

\subsection{Nonholonomic manifolds}

([VG1], [VG2]) Let $M^{n}$ be an $n$-dimensional manifold with a nonintegrable distribution $D$. Let

$$
D=D_{1} \subset D_{2} \subset D_{3} \cdots \subset D_{d}
$$

be the sequence of strict inclusions, where the fiber of $D_{i}$ at a point $x \in M$ is

$$
\left.\left.D_{i-1}\right|_{x} \cup\left[D_{1}, D_{i-1}\right]\right|_{x}
$$

(here $\left.\left[D_{1}, D_{i-1}\right]=\left\{[X, Y] \mid X \in D_{1}, Y \in D_{i-1}\right\}\right)$ and $d$ is the least number such that

$$
\left.\left.D_{d}\right|_{x} \cup\left[D_{1}, D_{d}\right]\right|_{x}=\left.D_{d}\right|_{x} .
$$

In case $D_{d}=T M$ the distribution is called completely nonholonomic. The number $d=d(M)$ is called the nonholonomicity degree. A manifold $M$ with a distribution $D$ on it will be referred to as nonholonomic one if $d(M) \neq 1$.

Here we will consider nonholonomic manifolds only when they are completely nonholonomic. This does not diminish generality, actually, since if the distribution in question is not completely nonholonomic, we can take the integral manifold corresponding to it; the restriction of the same distribution onto this integral manifold is completely nonholonomic, all right. If, nevertheless, we have to, for some reason, consider the general case it is easy mutatis mutandis; it corresponds to nontransitive Shchepochkina prolongs, cf. below, and the general machinery is easily adjusted to them).

Let $n_{i}(x)=\left.\operatorname{dim} D_{i}\right|_{x}$. The distribution $D$ is called a regular one if all the dimensions $n_{i}$ are constants on $M$. In what follows we will only consider regular distributions. This is also not a restriction if everything is smooth.

The tangent bundle over a nonholonomic manifold $(M, D)$ can be naturally endowed with a bundle of nilpotent Lie algebras as follows. Let $L$ be the Lie algebra of vector fields preserving the distribution in a neighborhood of some point $x \in M$. Naturally, $L$ is filtered and let $\mathfrak{g}$ be the associated graded Lie algebra. Set

$$
\mathfrak{g}_{-}=\underset{-d \leqslant i \leqslant-1}{\oplus} \mathfrak{g}_{i} .
$$

Clearly, $d$, the depth of $\mathfrak{g}_{-}$, coinsides with the nonholonomicity degree of $D$, see [VG2].

An analog of $G$-structure for nonholonomic manifolds is defined to be the pair $\left(\mathfrak{g}_{-}, \mathfrak{g}_{0}\right)$, where $\mathfrak{g}_{0}$ is a subalgebra of the Lie subalgebra which preserves the $\mathbb{Z}$ grading of $\mathfrak{g}_{-}$, i.e., $\mathfrak{g}_{0} \subset\left(\mathfrak{d} \mathfrak{e r} \mathfrak{g}_{-}\right)_{0}$. If $\mathfrak{g}_{0}$ is not indicated explicitely we assume that $\mathfrak{g}_{0}=\left(\mathfrak{d e r} \mathfrak{g}_{-}\right)_{0}$, i.e., is the largest one.

Thus, we see that with every nonholonomic manifold $(M, D)$ a natural $G$-structure is associated: $\operatorname{Lie}(G)=\left(\mathfrak{d e r} \mathfrak{g}_{-}\right)_{0}$. But the structure functions of this $G$-structure do 
not reflect the nonholonomic nature of $M$. Indeed, when we compute the structure functions (be they "traditional" $H_{\left(\mathfrak{g}_{-1}, \mathfrak{g}_{0}\right)_{*}}^{k, 2}$, or introduced above $H^{s}\left(\mathfrak{g}_{-1} ;\left(\mathfrak{g}_{-1}, \mathfrak{g}_{0}\right)_{*}\right)$ ) we use the fact that $\mathfrak{g}_{-1}$ is a commutative Lie algebra (partial derivatives commute).

To take the nonholonomic nature of $M$ into account we need an analog of the above Proposition for the case when the natural basis of the tangent space consists not of partial derivatives but rather of covariant derivatives corresponding to the connection determined by the same Pfaff forms whose vanishing determines the distribution. Therefore, instead of $\mathfrak{g}_{-1}$ we have a nilpotent algebra $\mathfrak{g}_{-}$.

So we have to define

(1) the simplest nonholonomic structure - the "flat" one, and

(2) the analog of Cartan prolong when $\mathfrak{g}_{-1}$ is replaced with $\mathfrak{g}_{-}$,

(3) (we have already understood) what is the analog of $\mathfrak{g}_{0}$ if it is not mentioned as is usually the case (only the distribution is given), but it remains to figure out

(4) what is the analog of $\left.\left(\mathfrak{g}_{-1}, \mathfrak{g}_{0}\right)_{*}\right)$, and, finally,

(5) what is the analog of $H_{\left(\mathfrak{g}_{-1}, \mathfrak{g}_{0}\right)_{*}}^{k, 2}$.

Let us answer these remaining questions.

Fix a frame $F$ in $\mathbb{K}^{n}$. Having identified $T_{v} V$ with $V$ by means of the transformation that preserves $D$, we fix a frame - the image of $F$ - in each $T_{v} V$. The nonholonomic structure of the nonholonomic manifold $\left(\mathbb{K}^{n}, D\right)$ is the pair of bundles: the frame bundle and the distribution $D$. The nonholonomic structure of $\left(\mathbb{K}^{n}, D\right)$ will be considered to be flat if the frames over $v$ are obtained from the fixed one by means of the $G$-action, where $G$ is a group whose Lie algebra is $\left.(\mathfrak{d e r} \mathfrak{g})_{-}\right)_{0}$.

\subsection{Structure functions of a nonholonomic manifold}

Given a pair $\left(\mathfrak{g}_{-}, \mathfrak{g}_{0}\right)$ as above, define its $k$-th Shchepochkina prolong (first published by I. Shchepochkina [Sh] for Lie superalgebras; see also more accessible [Sh5]) for $k>0$ to be:

$$
\mathfrak{g}_{k}=\left(i\left(S^{*}\left(\mathfrak{g}_{-}\right)^{*} \otimes \mathfrak{g}_{0}\right) \cap j\left(S^{*}\left(\mathfrak{g}_{-}\right)^{*} \otimes \mathfrak{g}_{-}\right)\right)_{k},
$$

where the subscript singles out the component of degree $k$ and where $i: S^{k+1}\left(\mathfrak{g}_{-1}\right)^{*} \otimes$ $\mathfrak{g}_{-1} \longrightarrow S^{k}\left(\mathfrak{g}_{-1}\right)^{*} \otimes \mathfrak{g}_{-1}^{*} \otimes \mathfrak{g}_{-1}$ is a natural embedding and $j: S^{k}\left(\mathfrak{g}_{-1}\right)^{*} \otimes \mathfrak{g}_{0} \longrightarrow$ $S^{k}\left(\mathfrak{g}_{-1}\right)^{*} \otimes \mathfrak{g}_{-1}^{*} \otimes \mathfrak{g}_{-1}$ a natural map.

Similarly to the case when $\mathfrak{g}_{-}$is commutative, define $\left(\mathfrak{g}_{-}, \mathfrak{g}_{0}\right)_{*}$ to be $\underset{k \geqslant-d}{\oplus} \mathfrak{g}_{k}$; then, as is easy to verify, $\left(\mathfrak{g}_{-}, \mathfrak{g}_{0}\right)_{*}$ is a Lie algebra. By the same reasons as for the manifolds with $G$-structure $([\mathbf{S t}])$, the space $H^{2}\left(\mathfrak{g}_{-} ;\left(\mathfrak{g}_{-}, \mathfrak{g}_{0}\right)_{*}\right)$ is the space of structure functions - obstructions to the identification of the infinitesimal neighborhood of a point on the manifold with a nonholonomic structure (given by $\mathfrak{g}_{-}$ and $\mathfrak{g}_{0}$ ) with same on a flat nonholonomic manifold with the same $\mathfrak{g}_{-}$and $\mathfrak{g}_{0}$.

The space $H^{2}\left(\mathfrak{g}_{-} ;\left(\mathfrak{g}_{-}, \mathfrak{g}_{0}\right)_{*}\right)$ naturally splits into homogeneous components whose degree will be called the order of the structure function; the minimal order of structure function is equal to $2-d$. As in the case of a commutative $\mathfrak{g}_{-}=\mathfrak{g}_{-1}$, the structure functions of order $k$ can be interpreted as obstructions to flatness of the nonholonomic manifold with the $\left(\mathfrak{g}_{-}, \mathfrak{g}_{0}\right)$-structure provided the obstructions of lesser orders vanish. Observe that for a nonholonomic manifold the order of structure functions is no more in direct relation with the numbers of the infinitesimal 
neighborhoods of the points we wish to identify: distinct partial derivatives bear different "weights".

When I told this to A. Beilinson in 1989 he winced and, as I got this far, he said that though he conceeded the idea is of interest, he doubted it can work in the general case: all my constructions are graded, whereas with every distribution only filtered algebras $\mathfrak{g}_{-}$are naturally associated. This incredulity is justified but fortunately, different filtrations on $\mathfrak{g}_{-}$with the same graded $\mathfrak{g}_{-}$are governed precisely by the structure functions of lesser orders and all of them must vanish for the N-curvature to be well-defined. So, given a filtered $\mathfrak{g}_{-}$, just take the associated graded algebra and proceed as indicated above. This indicates a way to study the stable normal forms of Pfaff equations listed in $[\mathbf{Z}]$.

\section{§3. The Lie algebra $\mathfrak{e}$ of Engel vector fields}

Let us describe the Lie algebra $\mathfrak{e}$ of vector fields that preserve the Engel distribution. Since each of the forms $\alpha_{1}$ and $\alpha_{2}$ is a familiar contact form, we know enough to demonstrate (we leave it as an easy exercise for the reader) the following statement.

Lemma . A weight basis of the $\mathbb{Z}$-graded Lie algebra $\mathfrak{e}$ is as follows (indicated are the degrees):

\begin{tabular}{|c|c|c|c|}
\hline-3 & -2 & -1 & 0 \\
\hline$\partial_{4}$ & $\partial_{3}+x_{1} \partial_{4}$ & $\partial_{1}$ & $x_{1} \partial_{1}+x_{2} \partial_{2}+2 x_{3} \partial_{3}+3 x_{4} \partial_{4}$ \\
& & $\partial_{2}+x_{1} \partial_{3}+\frac{1}{2} x_{1}^{2} \partial_{4}$ & $x_{1} \partial_{1}-x_{2} \partial_{2}+x_{4} \partial_{4}$ \\
& & & $x_{1} \partial_{2}+\frac{x_{1}^{2}}{2} \partial_{3}+\frac{x_{1}^{3}}{3} \partial_{4}$ \\
\hline
\end{tabular}

$$
\begin{array}{|c|}
\hline n \geqslant 1 \\
x_{1}^{n+1} \partial_{2}+\frac{x_{1}^{n+2}}{n+2} \partial_{3}+\frac{x_{1}^{n+3}}{n+3} \partial_{4} \\
\hline
\end{array}
$$

The structure of the Engel algebra $\mathfrak{e}$ seem to have never been described; so let us do it. First, introduce a shorthand for some elements of $\mathfrak{e}$ :

$$
\begin{aligned}
D_{3} & =\partial_{3}+x_{1} \partial_{4} ; \\
E & =x_{1} \partial_{1}+x_{2} \partial_{2}+2 x_{3} \partial_{3}+3 x_{4} \partial_{4} ; \\
H & =x_{1} \partial_{1}-x_{2} \partial_{2}+x_{4} \partial_{4} ; \\
X_{n} & =x_{1}^{n+1} \partial_{2}+\frac{x_{1}^{n+2}}{n+2} \partial_{3}+\frac{x_{1}^{n+3}}{n+3} \partial_{4} \quad \text { for } n \geqslant-1 .
\end{aligned}
$$

Now, it is clear that $\mathfrak{e}$ is solvable with

$$
\mathfrak{e} /[\mathfrak{e}, \mathfrak{e}]=\operatorname{Span}(E, H) ; \quad \mathfrak{e}^{(1)} /\left[\mathfrak{e}^{(1)}, \mathfrak{e}^{(1)}\right]=\operatorname{Span}\left(\partial_{1}\right), \text { where } \mathfrak{e}^{(1)}=[\mathfrak{e}, \mathfrak{e}] .
$$

The negative part, $\mathfrak{e}_{-}$plays for $\mathfrak{e}$ the same role the Heisenberg algebra plays for the contact algebra. This negative part was, of course calculated earlier, see, e.g., $[\mathbf{G 1}]$ where it also bears the name of Engel algebra; perhaps one should say the 
"small" and the "total" ones. It seems very strange that the total algebra had never beed desribed; I am sure such a description is buried in works of classics.

In review to [G1] [MR 96k:58010] it is written "There is a Darboux theorem for Engel structures: any two are locally diffeomorphic". Vershik also quoted this statement as a well-known. But I can not place it. Indeed, by Darboux theorem for the contact structure I understand the statement that says that the contact form can be locally reduced to a canonical form , cf. [Z]. (In contradistinction, a related statement that the nondegenerate differential 2 -form $\omega$ can only be reduced to a canonical form if and only if $d \omega=0$, cf. $[\mathbf{P}]$, so the statement on reducibility of the nondegenerate closed 2-form, sometimes encountered in textbooks on Differential Geometry, is a tautology.)

Concerning Engel structures, there is, for example, paper [G2] with examples of several non-equivalent Engel structures on $\mathbb{R}^{4}$. Doesn't it contradict the statement on an analog of Darboux theorem? So let us calculate the N-curvature of the contact and Engel structures: to find out if these invariants vanish, hence, there are analogs of Darboux theorem for them.

\section{§4. Structure functions of the contact and Engel structures}

Éli Cartan worked before "(co)homology" was defined, still, he introduced a notion that helps to calculate some of these cohomologies. This notion is involutive Lie algebra of vector fields. Serre gave a nice reformulation of this notion ([St]); thanks to Serre's statement for the case when $\left(\mathfrak{g}_{-}, \mathfrak{g}_{0}\right)_{*}$ is a simple vectorial Lie algebra instead of computing $H^{2}\left(\mathfrak{g}_{-1} ;\left(\mathfrak{g}_{-1}, \mathfrak{g}_{0}\right)_{*}\right)=\underset{k}{\oplus} H_{\left(\mathfrak{g}_{-1}, \mathfrak{g}_{0}\right)_{*}}^{k, 2}$, it only suffices to compute the summand with the least $k=1$ : only this term might be nonzero because $\left(\mathfrak{g}_{-}, \mathfrak{g}_{0}\right)_{*}$ is involutive. This really helps, see examples in [LPS].

Regrettably, Serre's theorem is only formulated so far for algebras of depth 1 . So I calculated $H^{2}\left(\mathfrak{g}_{-} ;\left(\mathfrak{g}_{-}, \mathfrak{g}_{0}\right)_{*}\right)$ by hands; later the result was confirmed by independent computer calculations up to convincingly high degree:

4.1. Theorem . The space of nonholonomic structure functions on any Engel manifold is of dimension 2. All structure functions are of order 2, their representatives are:

$$
\partial_{1}^{*} \wedge X_{-1}^{*} \otimes H+\partial_{1}^{*} \wedge X_{-1}^{*} \otimes E \text { and } \partial_{4}^{*} \wedge D_{3}^{*} \otimes \partial_{4} .
$$

The first of these cycles is "pure" in the sence that it does not represent its cohomology class modulo any coboundary, the coboundaries vanish. The other one is mixed: one can add any coboundary to it.

The following is another form of the classical theorem (see $[\mathbf{L P}]$ ):

4.2. Theorem . (Darboux theorem) The space of nonholonomic structure functions on any contact manifold vanishes. 


\section{References}

[A] Agrachev A., Methods of control theory in nonholonomic geometry. Proceedings of the International Congress of Mathematicians, Vol. 1, 2 (Zürich, 1994), 1473-1483, Birkhäuser, Basel, 1995

[BCG] Bryant R. L., Chern S. S., Gardner R. B., Goldschmidt H. L., Griffiths P. A., Exterior differential systems. Mathematical Sciences Research Institute Publications, 18. Springer-Verlag, New York, 1991. viii+475 pp.

[G1] Gershkovich V., On simplest Engel structures on 4-manifolds. In: R. P. Agarwal (ed.) Dynamical systems and applications, 279-294, World Sci. Ser. Appl. Anal., 4, World Sci. Publishing, River Edge, NJ, 1995

[G2] Gershkovich V., Exotic Engel structures on $\mathbb{R}^{4}$. Russian J. Math. Phys. 3 (1995), no. 2, 207-226

[G] Goncharov A., Infinitesimal structures related to hermitian symmetric spaces, Funct. Anal. Appl, 15, no. 3 (1981), 23-24 (Russian); a detailed version: id., Generalized conformal structures on manifolds. Selected translations. Selecta Math. Soviet. 6 (1987), no. 4, 307-340

[GL1] Grozman P., Leites D., Supergravities and $N$-extended Minkowski superspaces for any N. In: Wess J., Ivanov E. (eds.) Supersymmetries and quantum symmetries. Proc. International Conference in memory of V. Ogievetsky, June 1997, Lecture Notes in Physics 524, Springer, 1999, 58-67

[GL2] Grozman P., Leites D., Mathematica-aided study of Lie algebras and their cohomology. From supergravity to ballbearings and magnetic hydrodynamics. In: Keränen V. et al. (eds.) The second International Mathematica symposium, Rovaniemi, 1997, 185-192

[H] Hertz H., The principles of mechanics in new relation. NY, Dover, 1956

[L] Leites D. Supermanifold Theory. Karelia Branch of the USSR Acad. of Sci., Pertozavodsk, 1983, 200 pp. (in Russian; an enlarged version in English is available on the homepage matematik.su.se/ $\sim$ mleites)

[LPS] Leites D., Poletaeva E., Serganova V., On Einstein equations on manifolds and supermanifolds, J. Nonlinear Math. Physics, 2002, no. 2, (to appear)

[LP] Leites D., Poletaeva E., Supergravities and contact type structures on supermanifolds. Second International Conference on Algebra (Barnaul, 1991), 267-274, Contemp. Math., 184, Amer. Math. Soc., Providence, RI, 1995 
[LSV] Leites D., Serganova V., Vinel G., Classical superspaces and related structures. In: Bruzzo U. e.a. (eds.) Proc. Intnl. Conf. Diff. Geom. Methods in Physics DGM-IXI, 1990, Springer LN in Phys., 1991, 286-297

[M] Montgomery R., Engel deformations and contact structures. Northern California Symplectic Geometry Seminar, 103-117, Amer. Math. Soc. Transl. Ser. 2, 196, Amer. Math. Soc., Providence, RI, 1999

[P] Poletaeva E., Structure functions on the usual and exotic symplectic and periplectic supermanifolds. In: C. Bartocci, U. Bruzzo and R. Cianci (eds.) Differential geometric methods in theoretical physics (Rapallo, 1990), 390-395, Lecture Notes in Phys., 375, Springer, Berlin, 1991

[Sh] Shchepochkina I. Exceptional simple infinite dimensional Lie superalgebras, C.R. Acad. Sci. bulg. 1983, 36, n3, 313-314

[Sh5] Shchepochkina I., The five exceptional simple Lie superalgebras of vector fields, hep-th 9702120; id., The five exceptional simple Lie superalgebras of vector fields. Sov. J. Funct. Analisys, v.33, 1999, No.3, 14 pp.; id., Five exceptional simple Lie superalgebras of vector fields and their fourteen regradings. Representation Theory (electronic journal of AMS), v. 3, 1999, 3 (1999), 373-415

[St] Sternberg S. Lectures on differential geometry, 2nd ed, Chelsey, 1985

[T] Tanaka N., On infinitesimal automorphisms of Siegel domains, J. Math. Soc. Japan 22, 1970, 180-212; id., On the equivalence problems associated with simple graded Lie algebras. Hokkaido Math. J., 8 (1979), no. 1, 23-84

[V] Vershik, A. M. Classical and nonclassical dynamics with constraints. (Russian) In: Yu. G. Borisovich and Yu. E. Gliklikh (eds.) Geometry and topology in global nonlinear problems, 23-48, Novoe Global. Anal., Voronezh. Gos. Univ., Voronezh, 1984. English translation in: Yu. G. Borisovich and Yu. E. Gliklikh (eds.), Global analysis - studies and applications. I. A collection of articles translated from the Russian. Lecture Notes in Mathematics, 1108. Springer-Verlag, Berlin-New York, 1984, $278-301$

[VG1] Vershik A., Gershkovich V. An estimate of functional dimension of the space of orbits of germs of generic distributions. Math. USSR Zametki, 1988, 44: 5, 596-603

[VG2] Vershik A., Gershkovich V. Nonholonomic dinamical systems. In: Fundamental trends, VINITI, Moscow, 1987, 5-85 (translated by Springer in Sov. Math. Encyclop. series "Dynamical systems-7") 
[VG] Vershik, A. M.; Gershkovich, V. Ya. A bundle of nilpotent Lie algebras over a nonholonomic manifold (nilpotentization). (Russian) Zap. Nauchn. Sem. Leningrad. Otdel. Mat. Inst. Steklov. (LOMI) 172 (1989), Differentsialnaya Geom. Gruppy Li i Mekh. Vol. 10,21-40, 169 translation in J. Soviet Math. 59 (1992), no. 5, 1040-1053

[Z] Zhitomirskii, M., Typical singularities of differential 1-forms and Pfaffian equations. Translated from the Russian. Translations of Mathematical Monographs, 113. American Mathematical Society, Providence, RI; in cooperation with Mir Publishers, Moscow, 1992. xii+176 pp.

This article may be accessed via WWW at http://www.rmi.acnet.ge/hha/ or by anonymous ftp at

ftp://ftp.rmi.acnet.ge/pub/hha/volumes/2002/n2a17/v4n2a17.(dvi,ps,pdf)

Dimitry Leites mleites@matematik.su.se

Department of Mathematics, University of Stockholm,

Kräftriket hus 6, SE-106 91, Stockholm,

Sweden; 